\title{
BMJ Open Prognostic factors of idiopathic inflammatory myopathies complicated with interstitial lung disease: protocol for a systematic review and meta-analysis
}

\author{
Hiroyuki Kamiya, ${ }^{1}$ Ogee Mer Panlaqui, ${ }^{2}$ Shinyu Izumi, ${ }^{3}$ Takashi Sozu ${ }^{4}$
}

To cite: Kamiya $\mathrm{H}$, Panlaqui OM, Izumi S, et al. Prognostic factors of idiopathic inflammatory myopathies complicated with interstitial lung disease: protocol for a systematic review and meta-analysis. BMJ Open 2016;6:e012744. doi:10.1136/bmjopen-2016012744

- Prepublication history and additional material is available. To view please visit the journal (http://dx.doi.org/ 10.1136/bmjopen-2016012744).

Received 20 May 2016 Revised 17 October 2016 Accepted 18 October 2016

\section{CrossMark}

\footnotetext{
${ }^{1}$ Sakura Clinic, Chiba, Japan ${ }^{2}$ School of Public Health, University of Sydney, Sydney, Australia

${ }^{3}$ Department of Respiratory Medicine, National Center for Global Health and Medicine, Tokyo, Japan

${ }^{4}$ Faculty of Engineering, Department of Management Science, Tokyo University of Science, Tokyo, Japan
}

Correspondence to Dr Hiroyuki Kamiya; mlb04194@nifty.com

\section{ABSTRACT}

Introduction: Idiopathic inflammatory myopathies may be an overlapping disease complex. Although interstitial lung disease affects the mortality and the morbidity of the disease, a clinical course and the prognosis of the disease complicated with interstitial lung disease are diverse among individuals and prognostic factors have yet to be clarified. This article aims to report the rationale and the methodology of a future intended systematic review and meta-analysis of prognostic factors of idiopathic inflammatory myopathies complicated with interstitial lung disease. Methods and analysis: Participants are eligible if they are diagnosed as polymyositis/dermatomyositis, clinically amyopathic dermatomyositis or antisynthetase syndrome complicated with interstitial lung disease. Primary outcomes are all-cause and pulmonary-cause mortality and secondary outcomes include a progression of the disease and a deterioration of health-related quality of life. All primary studies of any design aside from case reports or case series are included. 2 reviewers will search electronic databases such as the MEDLINE, the EMBASE and the Science Citation Index Expanded and extract relevant data independently. A risk of bias in individual studies is evaluated based on the Quality in Prognostic Studies tool. Meta-analysis will be conducted if 3 or more studies are available for each outcome and pooled effects will be presented by the odds ratio $(O R)$. Where combining data is inappropriate due to a small number of studies or substantial heterogeneity, the result is reported qualitatively. Subgroup and sensitivity analysis are also considered based on clinical and methodological differences such as clinical manifestations, study designs and the quality of studies. The evidence level is assessed following the Grades of Recommendation, Assessment, Development and Evaluation (GRADE) method.

Ethics and dissemination: This study raises no ethical issues as it is based on the findings of previously published articles. The result will be reported in a peer-reviewed medical journal.

Trial registration number: CRD42016036999.

\section{Strengths and limitations of this study}

- A systematic review and meta-analysis of primary studies of any type of designs excluding case reports or case series to address a clinical question of prognosis.

- The first evidence based on a potentially large population derived from data synthesis for a rare disease.

- A potential difficulty in interpreting and applying the result due to diversity and a high risk of bias in included studies.

\section{INTRODUCTION}

\section{Aim of the report}

This article aims to report in detail the rationale and the methodology of an intended future systematic review and meta-analysis of prognostic factors of idiopathic inflammatory myopathies complicated with interstitial lung disease (ILD) to ensure rigorousness and transparency of the research. Any result expected to be derived from the review is not sought or presented in this report.

\section{Rationale}

ILD has been drawing much attention for the last few decades. ${ }^{1}$ It is partly because there is a growing number of patients with the disease due to the development of diagnostic tools ${ }^{2}$ and it is often difficult to be treated and can follow a fatal clinical course. $^{3}$ ILD is a comprehensive disease entity that demonstrates common final findings of parenchymal fibrosis mixed with inflammation despite a diversity of those mixtures among cases. ${ }^{4}$ While external stimuli such as a certain drug and an occupational exposure are noted to cause ILD, ${ }^{56}$ another 
notorious factor is connective tissue disease, which manifests ILD as a pulmonary complication. ${ }^{7}$

Polymyositis/dermatomyositis is one of the traditional connective tissue diseases and categorised into idiopathic inflammatory myopathies. ${ }^{8} 9$ It is triggered by unknown causes and progressed by an accelerated autoimmune reaction. ${ }^{10}$ Although polymyositis/dermatomyositis is characterised by proximal muscular weakness and unique cutaneous findings, ILD is frequently complicated and closely related to the morbidity and the mortality of the disease. ${ }^{11}$ Historically, anti-Jo-1 antibody, an autoantibody directed against histidyl-tRNA synthetase (one type of aminoacyl-tRNA synthetase (ARS)) in the cytoplasm, was identified in patients with polymyositis/ dermatomyositis and helped in the diagnosis of the disease as it is highly specific and predictive of the disease. ${ }^{12}$ The latest development in immunochemistry has discovered a large number of other autoantibodies that are also specific or associated with autoimmune myositis. ${ }^{13}$ In particular, the identification of anti-ARS antibodies other than anti-Jo-1 antibody is clinically important $^{14}$ and patients with those antibodies are noted to frequently present with cutaneous changes pathognomonic of dermatomyositis, arthralgia/arthritis and fever in addition to myositis and ILD. This led to the development of a new term called antisynthetase syndrome ${ }^{15}$ although manifestations of the disease could be diverse depending on the type of anti-ARS antibodies. ${ }^{16}$ Furthermore, anti-melanoma differentiationassociated gene 5 (anti-MDA5) antibody was identified in clinically amyopathic dermatomyositis, ${ }^{17}$ which is considered as a subgroup of dermatomyositis featuring clinically no or less muscular weakness and rapidlyprogressive ILD. ${ }^{18}$ It is recognised that the morbidity and the mortality of antisynthetase syndrome and clinically amyopathic dermatomyositis are also related to ILD. $^{1920}$

As polymyositis/dermatomyositis, clinically amyopathic dermatomyositis and antisynthetase syndrome demonstrate common findings regardless of some clinical differences, they may be on the same disease spectrum that characterises a complication of ILD, which will affect the prognosis of the disease. ${ }^{21}{ }^{22}$ However, it is generally believed that a clinical course is diverse and the prognosis varies among individuals although ILD is known to suggest a poor prognosis of the disease. ${ }^{23}{ }^{24}$ The identification of prognostic factors for patients with ILD will improve the management of this disease complex and provide great benefits within daily clinical practice as it will enable clinicians to predict the prognosis and implement medical resources effectively. There has been little literature available describing prognostic factors of this disease spectrum complicated with ILD and most currently available evidence is based on a small number of patients in a single or few medical institutions as this is a rare disease and thus could result in anecdotal reports. $^{25} 26$ Therefore, this systematic review has been planned to elucidate the prognostic factors of idiopathic inflammatory myopathies complicated with ILD and eventually to improve the prognosis of the disease.

\section{Hypothesis}

A clinical course of idiopathic inflammatory myopathies complicated with ILD is diverse and therefore there must be undefined factors related to the prognosis of the disease.

\section{Research question}

- What are the prognostic factors of idiopathic inflammatory myopathies complicated with ILD?

- What is the most predictive clinical information of the mortality of idiopathic inflammatory myopathies complicated with ILD?

- Is there any difference among prognostic factors of idiopathic inflammatory myopathies complicated with ILD depending on the difference of clinical manifestations (ie, polymyositis/dermatomyositis, clinically amyopathic dermatomyositis and antisynthetase syndrome)?

\section{Objective of the review}

This systematic review is intended to elucidate the prognostic factors of idiopathic inflammatory myopathies complicated with ILD and clarify what is the most predictive factor of the mortality of the disease.

\section{METHODS AND ANALYSIS}

\section{Registration and methodology}

This protocol is registered with PROSPERO (International Prospective Register of Systematic Reviews) at Centre for Review and Dissemination at University of York $^{27}$ (CRD42016036999) and reported following the guideline of PRISMA-P (Preferred Reporting Items for Systematic review and Meta-Analysis Protocols). ${ }^{28}$

\section{Timeline}

This study has yet to be initiated except for a pilot search and determining search terms and constructing a data extraction form. A full search was scheduled to be conducted in the first week of May 2016 and extended to the latest depending on the date of publication of this protocol.

\section{Eligibility criteria \\ Participants}

Patients with polymyositis/dermatomyositis, clinically amyopathic dermatomyositis and antisynthetase syndrome complicated with ILD of adult onset (over 16 years of age) are included. Polymyositis/dermatomyositis and clinically amyopathic dermatomyositis are diagnosed based on criteria such as Bohan and Peter ${ }^{8} 9$ and Sontheimer, ${ }^{29}$ which combine clinical, physiological and pathological findings as previously reported. Antisynthe syndrome is included if a complication of ILD is noted 
in addition to the positivity of anti-ARS antibody and another organ involvement such as myositis and unique cutaneous changes. The diagnosis of ILD is made based on physical examination, pulmonary function tests and radiological abnormalities. Patients are required to be followed up for at least 6 months. All patients are included at any time point during the disease course and from any clinical setting such as primary and secondary care. Juvenile myositis and overlap myositis are excluded from the review.

\section{Exposure or intervention (potential prognostic factors)}

All clinical information such as demographic features and disease profiles are considered as potential prognostic factors. A therapeutic intervention can also be a prognostic factor of the disease. Although there is no limitation as to the type of therapeutic interventions, only treatment with a duration of more than 6 months is a candidate for the prognostic factor. Comparators are no presence or fewer values of all of this information. Some studies may prespecify a prognostic factor of interest while others may only describe demographic, laboratory or radiological data depending on the occurrence of the outcome. Although the former case is obvious, all clinical information stated in the latter case is also sought to be analysed as potential prognostic factors.

\section{Outcomes and prioritisation}

All-cause and pulmonary-cause mortality are primary outcomes and secondary outcomes include a progression of the disease and a deterioration of health-related quality of life. The disease progression is defined based on the combined findings of symptomatic, functional (pulmonary function tests) and radiological changes over the follow-up period of time after the diagnosis or the initiation of treatment. An individual component comprising the combined criteria can also define a clinical course of the disease. Health-related quality of life is expected to be evaluated based on a questionnaire such as the 36-Item Short Form Health Survey (SF-36). ${ }^{30}$

\section{Studies}

Any type of primary studies excluding case reports or case series, whether prospective or retrospective, is included in the review if it describes the association of the predefined outcome with potential prognostic factors of polymyositis/dermatomyositis, clinically amyopathic dermatomyositis and antisynthetase syndrome with ILD. Unavailability of relevant statistics to describe the association does not exclude studies if they meet the inclusion criteria. If a study comprises a different subset of the disease complex, it is eligible for inclusion unless other ineligible cases such as juvenile myositis and overlap myositis are included. Editorials, letters and review articles are excluded. Although there is no limitation regarding the date of studies and the number of participants, studies are limited to the English literature. Conference proceedings with no further full reports and studies with only abstracts are also excluded due to concerns of lack of information unless detailed data are offered by authors.

\section{Information sources}

MEDLINE (via Ovid 1946-)

EMBASE (via Ovid 1974-)

Science Citation Index Expanded (via Web of Science 1900-)

Google Scholar

\section{Search strategy}

Two reviewers (HK/OMP) will search the Ovid MEDLINE and the Ovid EMBASE using key terms of study population and the methodology such as polymyositis, dermatomyositis, antisynthetase syndrome, ILD and prognosis. Appropriate search filters for prognostic studies of the MEDLINE and the EMBASE are derived from previous reports. ${ }^{31}{ }^{32}$ They are combined with both subject headings and text words of content-specific terms and their synonyms, which are determined referring to applicable reviews of the similar subject in the Cochrane Database of Systematic Reviews (see online supplementary appendix). Search terms are finalised through an independent attempt of construction and a pilot search by two reviewers and examining the agreement of retrieved articles. The Science Citation Index Expanded (via Web of Science) is also searched for citations, which are not covered by other electronic databases. In addition, review articles identified through the same search process over the past 5 years are screened and reference lists of relevant articles are also handsearched to identify potential primary articles. Authors of conference proceedings with no further full reports and studies with only abstracts are asked to provide relevant unpublished data. Grey literature is searched through Google Scholar following the previous report, ${ }^{33}$ which focuses on article titles of the first 300 reports using the aforementioned search terms. An expert in this field is also consulted to collect additional reports.

\section{Study records}

Data management

Relevant articles are managed through EndNote X7 and all extracted data are stored in a Microsoft Excel spreadsheet.

\section{Selection of studies and data extraction}

Two reviewers (HK/OMP) will independently examine titles and abstracts of all retrieved articles and select studies following the inclusion and exclusion criteria. If a duplicate or updated report is revealed, the study with the largest data set alone is included. However, multiple articles by the same research group are included if the outcome is different. Data are also extracted by the same reviewers (HK/OMP) based on the data extraction form, which has been predefined, reviewed and finalised through a pilot test to a small sample of eligible studies 
and a discussion among reviewers. A disagreement is resolved through a consultation with another reviewer.

\section{Data items}

The following data are extracted: name of the first author, the publication year, study location, study designs, follow-up periods, study population, pattern of ILD, the number of participants and their demographic features such as the age and the gender, autoantibodies, comparators if applicable, the clinical outcome, counts of the outcome, potential prognostic factors, methods for statistical analysis of the association of prognostic factors with the outcome, summary statistics and items associated with a risk of bias. Both unadjusted and adjusted measurements are drawn and adjusted factors are also extracted if available.

\section{Assessment of a risk of bias in individual studies}

A risk of bias in individual studies is assessed based on the Quality in Prognostic Studies (QUIPS) tool. ${ }^{34} 35$ Specifically, it contains six domains: study participation, study attrition, prognostic factor measurement, outcome measurement, study confounding, and statistical analysis and reporting. Each domain is rated as having a high, moderate or low risk of bias and the overall risk of bias of a study is evaluated by a total rating of all domains. For example, a study showing a low risk of bias in all domains is defined as having a low risk of bias.

\section{Statistical analysis}

\section{Dealing with missing data}

If summary statistics to address the association of potential prognostic factors with the outcome are not obtained directly, they are estimated using other relevant data. If it is unfeasible, authors are contacted and asked to provide these data.

\section{Measurement of the association}

Two major study designs to address the question of prognosis are cohort and case-control studies. In general, the former is summarised with the hazard ratio (HR) using the Cox proportional hazard regression model where time-to-event data are fitted although the risk ratio (RR) or the risk difference (RD) may also be used to estimate the proportion of an event. The latter type of studies calculates the OR using the logistic regression model where only point estimates of an event are considered. Therefore, the common measurement of the association of potential prognostic factors with an outcome will include the OR, the RR, the RD and the HR.

If the HR is not directly provided, it is recalculated from other information such as the log rank test and the Kaplan-Meier survival curve. ${ }^{36}{ }^{37}$ The OR may also be unavailable directly through the logistic regression model and only the comparison of potential prognostic factors between two groups with and without an event may be presented. In this case the OR is calculated manually based on counts of the outcome.
The RD is affected by the baseline risk of an event, which can be varied among studies and thus unfavourable in pooling data. The RR has an advantage over the $\mathrm{RD}$ regarding this issue and therefore the latter is converted to the former if the proportion of an outcome in two comparative groups is available. The OR can be approximated to the RR if an outcome is rare ${ }^{38}$ and the $\mathrm{HR}$ can also be approximated to the RR or the OR if the follow-up duration is short and the ratio of occurrence of an outcome in two comparative groups is small in addition to the condition that the outcome is rare. ${ }^{39}$ In fact, a recent study with a large sample size demonstrated that the survival rates at 1 and 5 years were $97 \%$ and $91 \%$ in polymyositis/dermatomyositis with ILD while they were $99 \%$ and $95 \%$ in those without ILD. ${ }^{40}$ As a result, the OR, the RR and the HR are assumed to be interchangeable and the OR will be used to summarise the association of potential prognostic factors with the outcome. The association is reported following the convention that the value of over one indicates an increased risk of the outcome, that is, the $\mathrm{OR}>1.0$ indicates an increased chance of death.

Where potential prognostic factors are continuous variables, the mean difference may be presented from the comparison of groups with and without an event. The mean difference is divided by the SD and converted to the standardised mean difference for further analysis of the association.

\section{Data synthesis}

The results across studies are pooled if the outcome data are available in three or more studies. Summary effects are sought to be presented as the OR with the assumption that the OR, the RR and the HR could be interchangeably approximated to each other under a specific condition. Accordingly, the OR and the HR of continuous variables are assumed to be representing the same effect measurement and can be combined together as the OR while those of categorical variables are assumed similarly and can undergo the same data handling. The standardised mean difference, which may be presented as the effect measurement of continuous variables, is combined by itself. When the standardised mean difference is estimated from the comparison of groups with and without an event, it is difficult to be combined with the OR due to the same variable, which will be estimated through the logistic regression model with a binary outcome. This assumed situation is different from a previously reported case where a continuous and binary outcome can be combined. ${ }^{41}$ When the median is presented for continuous variables instead of the mean, the latter is estimated from the former using the range and a sample size according to the previous report. ${ }^{42}$ Briefly, for a smaller sample the mean is recalculated by a sum of the smallest and largest value and twice the median, which is divided by four whereas it is approximated to the median if a sample size is larger than 25 . 
Unadjusted and adjusted estimates of the association are combined separately as it is reasonably expected that prognostic studies can be distorted by confounders and presenting summary statistics with adjustment in comparison with crude effects without adjustment are more likely to demonstrate a meaningful result. If more than one multivariable model with adjustment is available, the model with the best fit or with the most variables is selected. If the number of variables is the same in all models, the model with a factor of interest showing the most conservative result is selected.

Meta-analysis is conducted by a random-effect model ${ }^{43}$ considering that there should be some extent of variability among studies due to clinical and methodological differences. Data such as the logarithmic scale of the OR or the standardised mean difference and their SEs are combined by the inverse variance method using the statistical software, Review Manager (RevMan) V.5.3 (Copenhagen: The Nordic Cochrane Centre, The Cochrane Collaboration, 2014). Summary effects of each prognostic factor are estimated as the OR or the standardised mean difference with the $95 \%$ CI and $\tau^{2}$, which indicates between-study variances. The $95 \%$ prediction interval will also be calculated. ${ }^{44}$ Statistical significance is set at the $5 \%$ level. If meta-analysis is inappropriate due to few studies or concerns of substantial heterogeneity, the result is reported qualitatively. However, in a case where a study comprises a different subset of the disease, that is, polymyositis and dermatomyositis, pooling data is sought by contacting authors and asking them to provide data in each subset.

\section{Heterogeneity}

Heterogeneity is assessed statistically by the $\chi^{2}$ test and $\mathrm{I}^{2}$. Statistical significance is set at the $10 \%$ level because of low power of the test and the magnitude of heterogeneity is interpreted as not important $(0-30 \%)$, moderate $(30-50 \%)$, substantial $(50-70 \%)$ and considerable $(70-100 \%)$ (JPT Higgins, S Green, eds. Cochrane Handbook for Systematic Reviews of Interventions Version 5.1.0 (updated March 2011). The Cochrane Collaboration 2011. http://www handbook.cochrane. org). Clinical heterogeneity is assumed to be mainly derived from a different subset of the disease and types of ILD and autoantibodies among included studies while methodological heterogeneity is caused by a variety of study designs such as prospective or retrospective studies and diverse follow-up lengths, sample sizes and study locations. In particular, clinically amyopathic dermatomyositis, which is characterised by high probability of a complication of rapidly progressive ILD and the presence of anti-MDA5 antibody, may be a different group of the same spectrum of the disease. Therefore, subgroup analysis is considered if data are available according to the difference of clinical manifestations (polymyositis, dermatomyositis, clinically amyopathic dermatomyositis and antisynthetase syndrome) and types of ILD (acute or rapidly progressive and chronic) and autoantibodies identified (anti-ARS antibody including anti-Jo-1 antibody and non-Jo-1 antibody, and anti-MDA5 antibody). An analysis of studies with the same design such as a prospective cohort and a casecontrol study is also explored. In addition, the influence of different follow-up lengths is analysed based on two different time points; 1 and 5 years. Summary effects will also be presented as their original statistical forms, that is, the OR, the RR and the HR, to investigate the validity of considering these three statistics as interchangeable. The effect of a sample size and a study location on the outcome is sought to be revealed by dividing them into a few arbitrary groups, that is, $<50,50$ to 100 or over 100 and Asia or non-Asia, respectively. Sensitivity analysis is conducted focused on studies with a low risk of bias alone.

\section{Meta-biases}

Small study bias including publication bias is evaluated graphically examining the presence of asymmetry in a funnel plot and statistically by the Egger's test with the natural logarithmic scale of the OR being regressed against its SE if meta-analysis is based on 10 or more studies for an outcome. ${ }^{45}$ Statistical significance for asymmetry is set at the $10 \%$ level because of the low power of the test. If publication bias is statistically suspected, the number of missing studies and an adjusted summary effect are estimated by the method of trim and fill. ${ }^{46}$ Selective reporting is assessed examining the consistency of study findings with its protocol if available.

\section{Confidence in cumulative evidence}

It was reported that the Grades of Recommendation, Assessment, Development and Evaluation (GRADE) method could be useful in the assessment of prognostic reviews as in the case of assessing treatment effects. ${ }^{47}$ Although the report focused on the question of prognosis in a specific population rather than prognostic factors, five domains described to rate down the quality of evidence (risk of bias, inconsistency, imprecision, indirectness and publication bias) and two domains to rate it up (large effect and dose-response gradient) are applicable for this review as the fundamental methodological process of evaluation is similar between these two types of prognosis studies.

\section{ETHICS AND DISSEMINATION}

This systematic review is based on the summary result of previously published articles and individual patient data will not be obtained or accessed. Even if authors of included studies are asked to provide relevant missing data, any clinical information connecting with an individual patient will not be revealed. Therefore, there is no concerning ethical issue in the conduct of this research. The result of the review will be reported in a peer-reviewed medical journal following the PRISMA ${ }^{48}$ and the MOOSE (Meta-analysis of Observational Studies 
in Epidemiology) statement. ${ }^{49}$ Any information, which is obtained or used in the process of conducting the review, will be offered individually on request. The Microsoft Excel spreadsheet, which stores all data extracted from included studies and is the basis of the analysis in this research, may become open to the public in a digital repository such as Dryad after the final result is published in a journal.

\section{DISCUSSION}

This review is intended to elucidate prognostic factors of idiopathic inflammatory myopathies complicated with ILD and identify the most predictive factor of the mortality of this disease complex. Although some literature has addressed this clinical question, ${ }^{25} 26$ the reports are based on a small population in a few medical institutions. In addition, a systematic review has yet to be conducted to solve the issue. Therefore, this will be the first comprehensive review to answer the question and be a valuable guide for clinicians to treat patients with this diverse disease spectrum. Moreover, it will benefit patients from the appropriate medical care based on higher evidence and decrease the improper implementation of medical resources, which may eventually contribute to reduce the burden on the society.

However, there are some methodological limitations in the conduct of this review. First, conference proceedings and studies with only abstracts are excluded, which may lead to biased result although we believe that the influence of the issue can be reduced to the minimum by contacting authors and requesting them to offer the relevant data. Second, our statistical assumption that the $\mathrm{OR}$, the RR and the HR could be interchangeably approximated to each other may not necessarily be correct although this is partly supported by one of the largest studies, which reported that the mortality of idiopathic inflammatory myopathies complicated with ILD is not high. ${ }^{40}$ However, we also believe that this will not affect the validity of result because summary effects are also presented in their original statistical forms. Finally, it may be difficult to combine data from all eligible studies and estimate summary effects due to clinical and methodological diversity among studies, which may reduce statistical power in the analysis and diminish the significance of the review. However, we also expect this issue to be solved at least to some extent by requesting authors to offer relevant data. In addition, as the importance of a systematic review is not necessarily based on statistical data synthesis and qualitative analysis of result will remain meaningful, we believe that the value of the review will never be affected by this issue.

\section{CONCLUSIONS}

The rationale and the methodology of a systematic review and meta-analysis of prognostic factors of idiopathic inflammatory myopathies complicated with ILD were described. Although there are some methodological limitations in conducting the review, they will not be serious enough to affect its value. The result of the review is expected to be a future guide for both clinicians and patients to treat the disease.

Contributors HK conceived this research project and planned the entire methods to undertake it. He also wrote the manuscript of this protocol. OMP made contributions in conceiving this research project and planning literature search strategy and data extraction. SI was involved in the process of selecting the appropriate population and the outcomes targeted in this review. TS made contributions in planning statistical analysis, in particular, determining the appropriate statistical methods to report summary effects and data synthesis.

Competing interests None declared.

Provenance and peer review Not commissioned; externally peer reviewed.

Data sharing statement All data extracted from eligible studies are stored in a Microsoft Excel spreadsheet and the results will be produced based on the data on this sheet. This summary data will be provided to an individual researcher if requested and may also be made accessible to the public in a repository such as Dryad after the results of the study are published.

Open Access This is an Open Access article distributed in accordance with the Creative Commons Attribution Non Commercial (CC BY-NC 4.0) license, which permits others to distribute, remix, adapt, build upon this work noncommercially, and license their derivative works on different terms, provided the original work is properly cited and the use is non-commercial. See: http:/l creativecommons.org/licenses/by-nc/4.0/

\section{REFERENCES}

1. Raghu G, Rochwerg B, Zhang Y, et al. An Official ATS/ERS/JRS/ ALAT Clinical Practice Guideline: treatment of idiopathic pulmonary fibrosis. An Update of the 2011 Clinical Practice Guideline. Am J Respir Crit Care Med 2015;192:e3-19.

2. King TE Jr. Clinical advances in the diagnosis and therapy of the interstitial lung diseases. Am J Respir Crit Care Med 2005;172:268-79.

3. Ley B, Collard HR, King TE Jr. Clinical course and prediction of survival in idiopathic pulmonary fibrosis. Am J Respir Crit Care Med 2011;183:431-40.

4. Leslie KO. Historical perspective: a pathologic approach to the classification of idiopathic interstitial pneumonias. Chest 2005;128 (Suppl 1):513S-19S.

5. Kubo K, Azuma A, Kanazawa M, et al. Consensus statement for the diagnosis and treatment of drug-induced lung injuries. Respir Investig 2013;51:260-77.

6. Gulati M, Redlich CA. Asbestosis and environmental causes of usual interstitial pneumonia. Curr Opin Pulm Med 2015;21:193-200.

7. Fischer A, Lee JS, Cottin V. Interstitial lung disease evaluation: detecting connective tissue disease. Respiration 2015;90:177-84.

8. Bohan A, Peter JB. Polymyositis and dermatomyositis (first of two parts). N Engl J Med 1975;292:344-7.

9. Bohan A, Peter JB. Polymyositis and dermatomyositis (second of two parts). N Engl J Med 1975;292:403-7.

10. Casciola-Rosen L, Nagaraju K, Plotz P, et al. Enhanced autoantigen expression in regenerating muscle cells in idiopathic inflammatory myopathy. J Exp Med 2005;201:591-601.

11. Marie I, Hatron PY, Dominique S, et al. Short-term and long-term outcomes of interstitial lung disease in polymyositis and dermatomyositis: a series of 107 patients. Arthritis Rheum 2011;63:3439-47.

12. Yoshida S, Akizuki M, Mimori T, et al. The precipitating antibody to an acidic nuclear protein antigen, the Jo-1, in connective tissue diseases. A marker for a subset of polymyositis with interstitial pulmonary fibrosis. Arthritis Rheum 1983;26:604-11.

13. Love LA, Leff RL, Fraser DD, et al. A new approach to the classification of idiopathic inflammatory myopathy: myositis-specific autoantibodies define useful homogeneous patient groups. Medicine (Baltimore) 1991;70:360-74.

14. Nakashima R, Imura Y, Hosono Y, et al. The multicenter study of a new assay for simultaneous detection of multiple anti-aminoacyltRNA synthetases in myositis and interstitial pneumonia. PLOS ONE 2014;9:e85062. 
15. Marguerie C, Bunn CC, Beynon HL, et al. Polymyositis, pulmonary fibrosis and autoantibodies to aminoacyl-tRNA synthetase enzymes Q J Med 1990;77:1019-38.

16. Hamaguchi $Y$, Fujimoto $M$, Matsushita $T$, et al. Common and distinct clinical features in adult patients with anti-aminoacyl-tRNA synthetase antibodies: heterogeneity within the syndrome. PLOS ONE 2013;8: e60442.

17. Sato S, Hirakata M, Kuwana M, et al. Autoantibodies to a 140-kd polypeptide, CADM-140, in Japanese patients with clinically amyopathic dermatomyositis. Arthritis Rheum 2005;52:1571-6.

18. Gerami P, Schope JM, McDonald L, et al. A systematic review of adult-onset clinically amyopathic dermatomyositis (dermatomyositis sine myositis): a missing link within the spectrum of the idiopathic inflammatory myopathies. J Am Acad Dermatol 2006;54:597-613.

19. Yoshifuji H, Fujii T, Kobayashi S, et al. Anti-aminoacyl-tRNA synthetase antibodies in clinical course prediction of interstitial lung disease complicated with idiopathic inflammatory myopathies. Autoimmunity 2006;39:233-41.

20. Ye S, Chen XX, Lu XY, et al. Adult clinically amyopathic dermatomyositis with rapid progressive interstitial lung disease: a retrospective cohort study. Clin Rheumatol 2007;26:1647-54.

21. Yamasaki $\mathrm{Y}$, Yamada $\mathrm{H}$, Ohkubo $\mathrm{M}$, et al. Longterm survival and associated risk factors in patients with adult-onset idiopathic inflammatory myopathies and amyopathic dermatomyositis: experience in a single institute in Japan. J Rheumatol 2011;38:1636-43.

22. Marie I, Josse S, Hatron PY, et al. Interstitial lung disease in anti-Jo-1 patients with antisynthetase syndrome. Arthritis Care Res 2013;65:800-8.

23. Hervier B, Benveniste O. Clinical heterogeneity and outcomes of antisynthetase syndrome. Curr Rheumatol Rep 2013;15:349.

24. Hervier B, Devilliers H, Stanciu R, et al. Hierarchical cluster and survival analyses of antisynthetase syndrome: phenotype and outcome are correlated with anti-tRNA synthetase antibody specificity. Autoimmun Rev 2012;12:210-17.

25. Fujisawa $\mathrm{T}$, Hozumi $\mathrm{H}$, Kono $\mathrm{M}$, et al. Prognostic factors for myositisassociated interstitial lung disease. PLOS ONE 2014;9:e98824.

26. Hozumi H, Enomoto $\mathrm{N}$, Kono $\mathrm{M}$, et al. Prognostic significance of antiaminoacyl-tRNA synthetase antibodies in polymyositis/dermatomyositisassociated interstitial lung disease: a retrospective case control study. PLOS ONE 2015;10:e0120313.

27. Center for Review and Dissemination, University of York. PROSPERO International Prospective Register of Systematic Reviews. http://www. crd.york.ac.uk/prospero/(accessed 17 Apr 2016).

28. Shamseer L, Moher D, Clarke M, et al. Preferred reporting items for systematic review and meta-analysis protocols (PRISMA-P) 2015: elaboration and explanation. BMJ 2015;349:g7647.

29. Sontheimer RD. Would a new name hasten the acceptance of amyopathic dermatomyositis (dermatomyositis sine myositis) as a distinctive subset within the idiopathic inflammatory dermatomyopathies spectrum of clinical illness? J Am Acad Dermatol 2002;46:626-36.

30. Wilczynski NL, Haynes RB. Developing optimal search strategies for detecting clinically sound prognostic studies in MEDLINE: an analytic survey. BMC Med 2004;2:23.
31. Wilczynski NL, Haynes RB. Optimal search strategies for detecting clinically sound prognostic studies in EMBASE: an analytic survey. J Am Med Inform Assoc 2005;12:481-5.

32. Ware JE Jr, Snow KK, Kosinski M, et al. SF-36 Health Survey Manual and Interpretation Guide. Boston, MA: The Health Institute, New England Medical Center, 1993.

33. Haddaway NR, Collins AM, Coughlin D, et al. The role of Google Scholar in evidence reviews and its applicability to grey literature searching. PLOS ONE 2015;10:e0138237.

34. Hayden JA, Côté $P$, Bombardier $C$. Evaluation of the quality of prognosis studies in systematic reviews. Ann Intern Med 2006;144:427-37.

35. Hayden JA, van der Windt DA, Cartwright JL, et al. Assessing bias in studies of prognostic factors. Ann Intern Med 2013;158:280-6.

36. Parmar MK, Torri V, Stewart L. Extracting summary statistics to perform meta-analyses of the published literature for survival endpoints. Stat Med 1998;17:2815-34.

37. Tierney JF, Stewart LA, Ghersi D, et al. Practical methods for incorporating summary time-to-event data into meta-analysis. Trials 2007;8:16.

38. Deeks JJ, Altman DG. Effect measures for meta-analysis of trials with binary outcomes. In: Egger M, Smith GD, Altman DG, eds. Systematic reviews in health care meta-analysis in context. 2nd edn London: BMJ Publishing Groups, 2001:313-35.

39. Symons MJ, Moore DT. Hazard rate ratio and prospective epidemiological studies. J Clin Epidemiol 2002;55:893-9.

40. Johnson C, Pinal-Fernandez I, Parikh R, et al. Assessment of mortality in autoimmune myositis with and without associated interstitial lung disease. Lung 2016;194:733-7.

41. Whitehead A, Bailey AJ, Elbourne D. Combining summaries of binary outcomes with those of continuous outcomes in a meta-analysis. $J$ Biopharm Stat 1999:9:1-16.

42. Hozo SP, Djulbegovic B, Hozo I. Estimating the mean and variance from the median, range, and the size of a sample. BMC Med Res Methodol 2005:5:13.

43. DerSimonian R, Laird N. Meta-analysis in clinical trials. Control Clin Trials 1986;7:177-88.

44. Riley RD, Higgins JP, Deeks JJ. Interpretation of random effects meta-analyses. BMJ 2011;342:d549.

45. Egger M, Smith G, Schneider M, et al. Bias in meta-analysis detected by a simple, graphical test. BMJ 1997;315:629-34.

46. Duval S, Tweedie R. Trim and fill: a simple funnel-plot-based method of testing and adjusting for publication bias in meta-analysis. Biometrics 2000;56:455-63.

47. Iorio A, Spencer FA, Falavigna M, et al. Use of GRADE for assessment of evidence about prognosis: rating confidence in estimates of event rates in broad categories of patients. BMJ 2015;350:h870.

48. Moher D, Liberati A, Tetzlaff J, et al. Preferred reporting items for systematic reviews and meta-analyses: the PRISMA statement. Ann Intern Med 2009;151:264-9, w64.

49. Stroup DF, Berlin JA, Morton SC, et al. Meta-analysis of observational studies in epidemiology: a proposal for reporting. Meta-analysis Of Observational Studies in Epidemiology (MOOSE) group. JAMA 2000;283:2008-12. 